
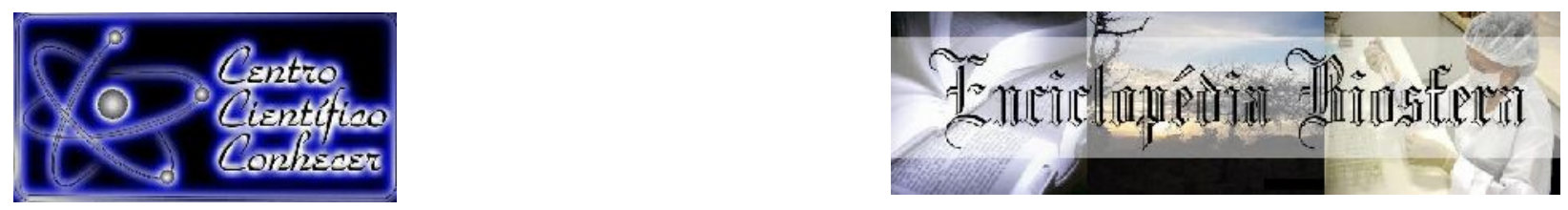

\title{
NOVA POLÍTICA NACIONAL DE ATENÇÃO BÁSICA (PNAB): VISÃO DOS PROFISSIONAIS DA ATENÇÃO BÁSICA DO MUNICÍPIO DE SÃO MATEUS-MA
}

\author{
Joelane Carvalho da Fonseca', Renato Sousa Mesquita', Patrícia Vieira de Oliveira ${ }^{2}$, \\ Wellyson da Cunha Araújo Firmo ${ }^{3}$, Alessandra Gonçalves Martins ${ }^{4^{*}}$ \\ ${ }^{1}$ Enfermeiro(a) pela Faculdade de Educação de Bacabal-FEBAC, Bacabal-MA, Brasil \\ ${ }^{2}$ Acadêmica de Biomedicina pela Universidade Ceuma, São Luís-MA, Brasil \\ ${ }^{3}$ Docente da Universidade Ceuma-UniCeuma, São Luís-MA, Brasil \\ ${ }^{4}$ Docente da Faculdade Pitágoras de Bacabal, Bacabal-MA, Brasil \\ *E-mail: martins_alessandra78@hotmail.com
}

Recebido em: 04/10/2019 - Aprovado em: 30/11/2019 - Publicado em: 15/12/2019

DOI: 10.18677/EnciBio_2019B40

\begin{abstract}
O Ministério da Saúde conceitua a Atenção Básica (AB) como um conjunto de ações de saúde individuais, familiares e coletivas que envolvem promoção, prevenção e proteção à saúde. A última reformulação da Política Nacional de Atenção Básica (PNAB) traz uma série de mudanças, sendo que muitas destas geraram uma série de questionamentos contrários e a favor da nova política. O objetivo desse estudo foi analisar a visão dos profissionais da $A B$ no município de São Mateus do MaranhãoMA, sobre a nova PNAB. Trata-se de um estudo descritivo, transversal, com abordagem quantitativa. Com entrevista de 40 profissionais de saúde da Estratégia Saúde da Família que trabalhavam nas Unidades Básica de Saúde. A coleta de dados foi feita no período de setembro e outubro de 2018, sendo análise de dados feita através do programa Microsoft Office Word $\AA$ e Excel® 2013. Os resultados demonstram que a maioria dos profissionais que participaram da pesquisa, $35 \%$ eram Agentes Comunitários de Saúde, $45 \%$ dos profissionais não tinham nenhum conhecimento a respeito das mudanças estabelecidas pela nova PNAB e $83 \%$ relatam ser um retrocesso as Equipe de Atenção Básica sem a obrigatoriedade de alguns profissionais. Sendo possível assim concluir, que diante de tamanhas mudanças, é esperado que dificuldades e dúvidas surjam, sendo que, a validação destes princípios dependerão da redução destas dificuldades, principalmente quando se trata do conhecimento dos profissionais em relação as novas políticas.
\end{abstract}

RESUMO

PALAVRAS-CHAVE: atenção básica, política, saúde coletiva.

\section{NEW NATIONAL POLICY ON BASIC CARE: VISION OF PRIMARY CARE PROFESSIONALS IN THE MUNICIPALITY OF SÃO MATEUS-MA.}

\begin{abstract}
Ministry of Health conceptualizes Primary Care $(\mathrm{PH})$ as a set of individual, family and collective health actions that involve health promotion, prevention and protection. The last reformulation of the National Policy of Primary Care (NPPC) brings a series of changes, many of which generated a series of opposing and in
\end{abstract}


favor of the new policy. The objective of this study was to analyze the view of the professionals of the $\mathrm{PH}$ in the city of São Mateus do Maranhão-MA, about the new NPPC. This is a descriptive cross-sectional study with a quantitative approach. 40 health professionals from the Family Health Strategy who worked in the Basic Health Units were interviewed. Data were collected from September to October 2018, and data were analyzed using Microsoft Office Word® and Exce|®. 2013. The results show that most of the professionals who participated in the survey, $35 \%$ were Community Health Agents, $45 \%$ of the professionals had no knowledge about the changes established by the new NPPC and $83 \%$ reported being a setback. Basic without the obligation of some professionals. Thus, it is possible to conclude that in the face of such changes, it is expected that difficulties and doubts arise, and the validation of these principles will depend on the reduction of these difficulties, especially when it comes to the knowledge of professionals regarding the new policies.

KEYWORDS: primary care, politics, public health.

\section{INTRODUÇÃO}

A Atenção Básica $(A B)$ ou Atenção Primária à Saúde (APS) tem seus fundamentos instituídos na conferência internacional sobre cuidados primários de Saúde, realizada no período de 06 a 12 de setembro de 1978, na cidade de AlmaAta quando foi realizada a Declaração de Alma-Ata. Essa importante declaração trouxe um conceito mais amplo de saúde, com uma meta de promovê-la a todos os povos até o ano 2.000, tendo como principal estratégia de alcance desta, os cuidados primários de saúde. Trouxe ainda um importante conceito do que seria a Atenção Primária (AP), considerando esta como o primeiro nível de contato dos indivíduos, da família e da comunidade com o serviço nacional de saúde, já se destacando o caráter preventivo (DECLARAÇÃO DE ALMA-ATA, 2001).

Seguindo estes pressupostos, o Brasil criou na década de 90 o Programa Saúde da Família (PSF), hoje conhecido como Estratégia Saúde da Família (ESF), que veio a se tornar a principal estratégia de ampliação e consolidação da $A B$ no Brasil, alcançando importantes índices de cobertura da população brasileira, sendo esse resultado impulsionado em parte, pelo seu papel prioritário na Política Nacional de Atenção Básica (PNAB), um importante documento que fixou as diretrizes operacionais para os três níveis de governo: municipal, estadual e federal (MOROSINI et al., 2018).

O Ministério da Saúde (MS) conceitua a $A B$ como um conjunto de ações de saúde individuais, familiares e coletivas que envolvem promoção, prevenção, proteção, diagnóstico, tratamento, reabilitação, redução de danos, cuidados paliativos e vigilância em saúde, desenvolvida por meio de práticas de cuidado integrado e gestão qualificada, realizada com equipe multiprofissional e dirigida à população em território definido, sobre as quais as equipes assumem responsabilidade sanitária (BRASIL, 2017a).

A PNAB foi instituída a princípio pela Portaria GM o 648 de 28 de março de 2006, sendo reformulada pela portaria GM no 2.488 de 21 de outubro de 2011 e recentemente pela portaria GM n 2.436 de 21 de setembro de 2017 (BRASIL, 2006; BRASIL, 2011; BRASIL, 2017a).

O Conselho Nacional de Saúde (CNS), antes mesmo da publicação da Portaria 2.436, na sexagésima primeira reunião extraordinária, realizada no dia 9 de agosto de 2017, aprovou a recomendação no 035, direcionada ao Congresso 
Nacional, MS, Conselho Nacional de Secretarias Municipais de Saúde (CONASEMS), Conselho Nacional de Secretários de Saúde (CONASS), Conselhos Estaduais e Municipais de Saúde e as entidades que compõem as instâncias de controle social do Sistema Único de Saúde (SUS), que não deliberasse sobre o processo de revisão da PNAB enquanto não houvesse exauridos todos os debates acerca deste documento (BRASIL, 2017b).

A última reformulação da PNAB trouxe uma série de mudanças nesta política, tais como: o reconhecimento das equipes de $A B$, sem a obrigatoriedade dos profissionais Agentes Comunitários de Saúde (ACS), dentistas e auxiliares ou técnicos de Enfermagem, a ampliação das atividades dos ACS, a figura do gerente de $A B$, a possibilidade do usuário se vincular a mais de uma Unidade Básica de Saúde (UBS), a possiblidade de formar equipes de saúde da família e equipes de atenção básica com população adscrita a partir de 2.000 pessoas ou menos dentro do território, sendo que muitas destas geraram uma série de questionamentos contrários, advindos de organizações como: Conselho Nacional de Saúde (CNS), Conselho Federal de Enfermagem (COFEN), Confederação Nacional dos Agentes Comunitários de Saúde (CONACS), dentre outras (BATALHA; LAVOR, 2017).

Apesar dos questionamentos contrários, para o CONASEMS e do MS, tais mudanças representam avanços significativos na forma de organização da $A B$ nos municípios brasileiros, conferindo a esses mais autonomia na gestão dos serviços básicos de saúde (MATHIAS, 2017).

Este estudo se fez necessário devido à importância da PNAB, que caracteriza a $A B$ como a principal porta de entrada para o centro de comunicação das Redes de Atenção à Saúde (RAS) no SUS. Sendo de grande relevância identificar qual a visão que tem os profissionais de saúde atuantes neste sistema sobre as mudanças contidas nesta nova política. Em virtude de tais questionamentos acerca das mudanças implantadas por meio da nova PNAB, o presente estudo teve como objetivo, analisar a visão dos profissionais da $A B$ no município de São Mateus do Maranhão-MA, sobre a nova PNAB.

\section{MATERIAL E MÉTODOS}

A pesquisa tratou-se de um estudo do tipo descritivo, transversal, com abordagem quantitativa. Foi realizada em cinco UBS da zona urbana do município de São Mateus do Maranhão-MA, sendo: UBS Avenida Piqui, São Marcos, Dr. Antônio Francisco da Costa Vage, Saraguá e Francisco Torres Paula Filho. O município encontra-se à cerca de $180 \mathrm{Km}$ da capital de São Luís e com estimativa de 41.350 habitantes (IBGE, 2018).

A amostra da pesquisa foi com os profissionais de saúde da ESF, incluindo os profissionais da saúde bucal, os ACS, dentistas, enfermeiros, médicos e técnicos de Enfermagem, que trabalhavam nas UBS, quando foi realizada a aplicação de 40 questionários, nos períodos da manhã e tarde, de segunda a sexta, com critérios de inclusão da pesquisa: profissionais de saúde que trabalhavam nos locais e que estavam presentes nos dias da aplicação do questionário.

A pesquisa ocorreu no período dos meses de setembro a outubro de 2018, quando foram aplicados os questionários com perguntas abertas e fechadas, entre as quais faziam questionamentos sobre informações socioeconômicas, sobre a visão dos profissionais a respeito da nova PNAB. Os dados foram analisados e agrupados em gráficos e tabelas, utilizando os Programas Microsoft Office Word® e 
Excel® 2013. O trabalho seguiu os preceitos éticos estabelecidos pela Resolução $466 / 2012$ do CNS.

\section{RESULTADOS E DISCUSSÃO}

Foram entrevistados 40 profissionais da saúde, todos trabalhadores nas UBS, da amostra 70\% são mulheres. A maioria dos entrevistados (56\%) estão entre 20 e 40 anos, $58 \%$ declararam-se de cor parda, $63 \%$ recebem renda entre um e dois salários mínimos, a maioria (63\%) são casados (Tabela 1)

TABELA 1- Distribuição numérica e percentual de variáveis sociais e demográficas dos participantes da pesquisa. São Mateus do Maranhão, Maranhão, Brasil, 2018.

\begin{tabular}{ccc}
\hline Variáveis & № & $\%$ \\
\hline Sexo & 28 & $70,0 \%$ \\
Feminino & 12 & $30,0 \%$ \\
Masculino & 40 & $100 \%$ \\
Total & & \\
Faixa etária & 11 & $28,0 \%$ \\
$20-30$ anos & 11 & $28,0 \%$ \\
$30-40$ anos & 8 & $20,0 \%$ \\
$40-50$ anos & 5 & $13,0 \%$ \\
$50-60$ anos & 5 & $13,0 \%$ \\
$60-70$ anos & 40 & $100 \%$ \\
Total & & \\
Etnia & 15 & $38,0 \%$ \\
Branca & 23 & $58,0 \%$ \\
Parda & 2 & $5,0 \%$ \\
Negra & 40 & $100 \%$ \\
Total & & \\
Renda salarial & 25 & $63,0 \%$ \\
1 a 2 salários & 11 & $28,0 \%$ \\
2 a 5 salários & 4 & $10,0 \%$ \\
5 a 10 salários & 40 & $100 \%$ \\
Total & & \\
Estado civil & 25 & $63,0 \%$ \\
Casado & 12 & $30,0 \%$ \\
Solteiro & 1 & $5,0 \%$ \\
Viúvo & 2 & $3,0 \%$ \\
União Consensual & 40 & $100 \%$ \\
Total & &
\end{tabular}

Fonte: Dados da pesquisa (2018).

A maioria dos entrevistados são mulheres, o que corrobora com pesquisa realizada por Santos (2017) que também encontrou maior número de mulheres trabalhando em UBS, a presença feminina nesta categoria profissional pode ser explicada pela característica das profissões voltadas para a área da saúde, ser compostas principalmente por este gênero.

O estudo de Velloso e Varanda (2017) assemelha-se ao presente trabalho, visto que encontraram a idade média dos profissionais de saúde entrevistados de 35 
anos, sendo não tão mais jovem, o que isso pode contribuir para a profissão, quando se pensa no quesito responsabilidade e compromisso desses profissionais.

A diversidade entre os salários se mostrou bem díspar, já que a maioria dos entrevistados possuía uma renda salarial entre 1 a 2 salários mínimos e apenas um grupo bem seleto de 10,0\% afirmaram receberem de 5 a 10 salários mínimos, essa diferença salarial, esteve associada principalmente, a formação, qualificação e alguns por terem cargos de gestão/confiança.

Dados semelhantes ao estudo atual são amplos, já que as diferenças de salário entre os membros de uma equipe de saúde são significativas, mostrado também por Santos et al. (2011) que revelam que apesar da importância imprescindível que todos os profissionais de saúde assumem dentro de uma equipe, incluindo o acúmulo de trabalho e responsabilidade, é divergente a diferença salarial entre os membros da mesma. Seguindo o mesmo estudo, expuseram ainda que a renda salarial dos ACS foi de menos de um salário mínimo, enquanto que os salários dos médicos diversificavam entre $R \$ 4.000,00$ a $8.000,00$, fator este que desestimulavam muitos profissionais participantes da pesquisa.

TABELA 2 - Distribuição numérica e percentual quanto a formação dos profissionais participantes da pesquisa. São Mateus do Maranhão, Maranhão, Brasil, 2018.

\begin{tabular}{ccc}
\hline Profissão & № & $\%$ \\
\hline Agentes Comunitários de Saúde & 14 & $35,0 \%$ \\
Dentista & 7 & $18,0 \%$ \\
Enfermeiro & 10 & $25,0 \%$ \\
Médico & 4 & $10,0 \%$ \\
Técnico de Enfermagem & 5 & $13,0 \%$ \\
Total & 40 & $100 \%$ \\
\hline
\end{tabular}

Fonte: Dados da pesquisa (2018).

Com referência aos profissionais de saúde que participaram da pesquisa, o estudo mostrou que a maioria dos entrevistados eram ACS $(35,0 \%)$, seguidos de enfermeiros (25,0\%), dentistas (18,0\%), técnicos de Enfermagem $(13,0 \%)$ e a minoria foi a classe médica com apenas $10,0 \%$.

Para a estabilização da ESF é necessária a existência de equipe multiprofissional, denominada ESF, integrada por médico generalista ou especialista em saúde da família, enfermeiro generalista ou também especialista em saúde da família, técnico de Enfermagem e ACS, podendo ser complementada como parte da equipe, os profissionais de saúde bucal, como os cirurgiões dentistas e auxiliares ou técnico em saúde bucal.

A ESF se firma em UBS que são responsáveis em atender uma determinada população anexada, sendo que cada uma apresenta responsabilidade sanitária por seu território de referência, sendo que o método de trabalho dos profissionais deve ser instruído pelo desenvolvimento do acesso, consolidação da relação, continuidade, coordenação e extensão do cuidado (GEUS et al., 2011).

A medida que ocorreram expansões em base municipal e seus atuais resultados a ESF obteve mais destaque. Dessa maneira, ganhou a posição de estratégia prioritária nos métodos organizativos da $A B$, se tornando o ponto central de investimento no MS que visa o fortalecimento do SUS no país (SAVASSI, 2012). 


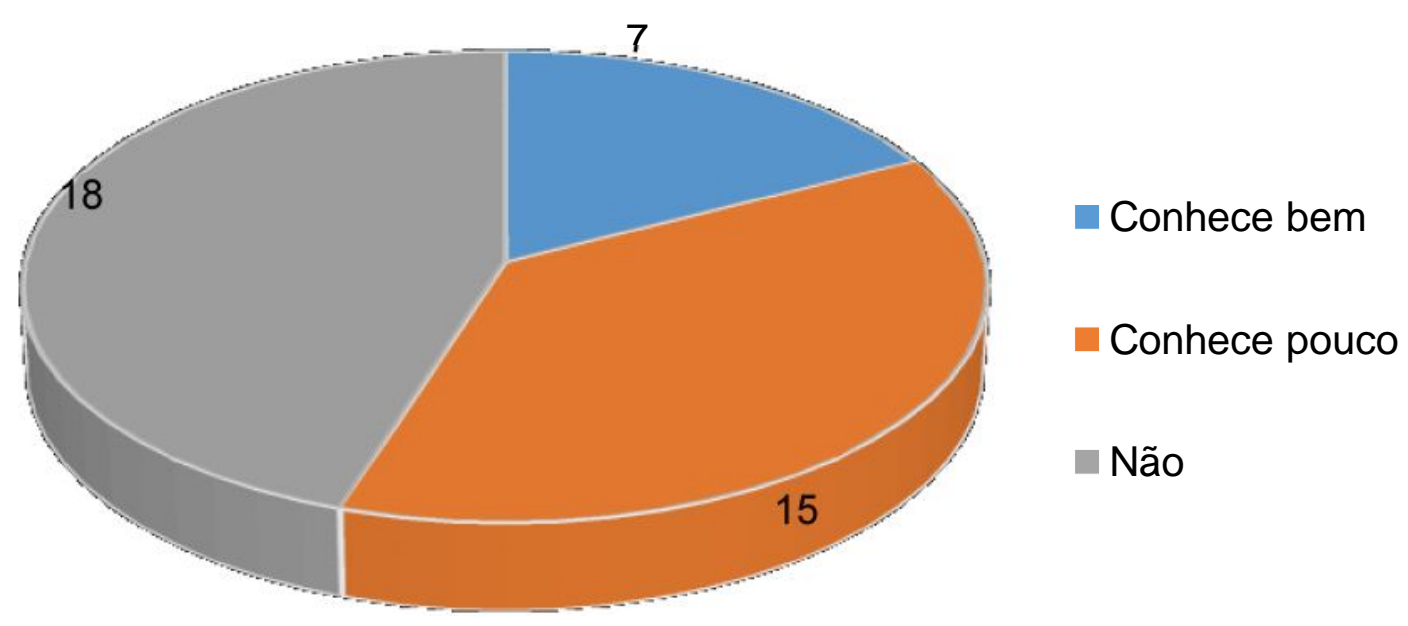

GRÁFICO 1 - Distribuição percentual sobre o conhecimento dos profissionais participantes da pesquisa a respeito da nova Política Nacional de Atenção Básica. São Mateus do Maranhão, Maranhão, Brasil, 2018.

Fonte: Dados da pesquisa (2018).

A respeito dos profissionais que tinham algum entendimento sobre a nova PNAB e consequentemente sobre as novas atribuições e mudanças, apenas $18,0 \%$ dos profissionais entrevistados afirmaram que conheciam bem acerca do assunto, $38,0 \%$ disseram que conheciam um pouco sobre a nova política e a maioria dos profissionais $(45,0 \%)$ revelaram que não conheciam nada a respeito do assunto.

Em setembro de 2017, foi publicada uma nova PNAB, que resumiu a crítica de organizações historicamente ligadas a defesa do SUS, como a Associação Brasileira de Saúde Coletiva (ABRASCO), Centro Brasileiro de Estudos de Saúde (CEBES) e a Escola Nacional de Saúde Pública (ENSP). Em um comunicado composto, as três instituições denunciaram a revogação da prioridade dada a ESF na organização do SUS com a possível perda de auxílios para outras composições da $A B$, com explicação em uma provável solução na área financeira da saúde. Com tudo isso, levou-se a apresentação de apreensão com retrocessos a respeito da construção de uma APS integral, que vinha conduzindo o modelo de $A B$ apoiado na ESF. Com todas as propostas divulgadas pela nova PNAB, abriu-se um leque de dúvidas, preocupações e benefícios por parte dos profissionais de saúde, quanto aos processos de integrações, falta de obrigatoriedade de alguns profissionais na equipe, possibilidade de inserção de gerentes na $A B$, assim como a possibilidade dos profissionais de se vincularem a mais de uma UBS, entre outras, gerou-se um grau de desarranjo na inclusão do profissional nestas mudanças, como pode ser visto no estudo atual, a maioria dos profissionais não tinham concepções exatas em relação a tais transfigurações (ABRASCO et al., 2017).

Em um estudo feito por Santos (2017) quando foram entrevistados 94 profissionais em nove municípios, foi constatado que os profissionais participantes da pesquisa também tinham deficiência de conhecimento em relação as novas políticas, relatando a dificuldade em acompanhar as transições apresentadas em 
muitas atividades atualizadas da AP ou que ainda estão em processo de legitimação com evidentes mudanças institucionais.

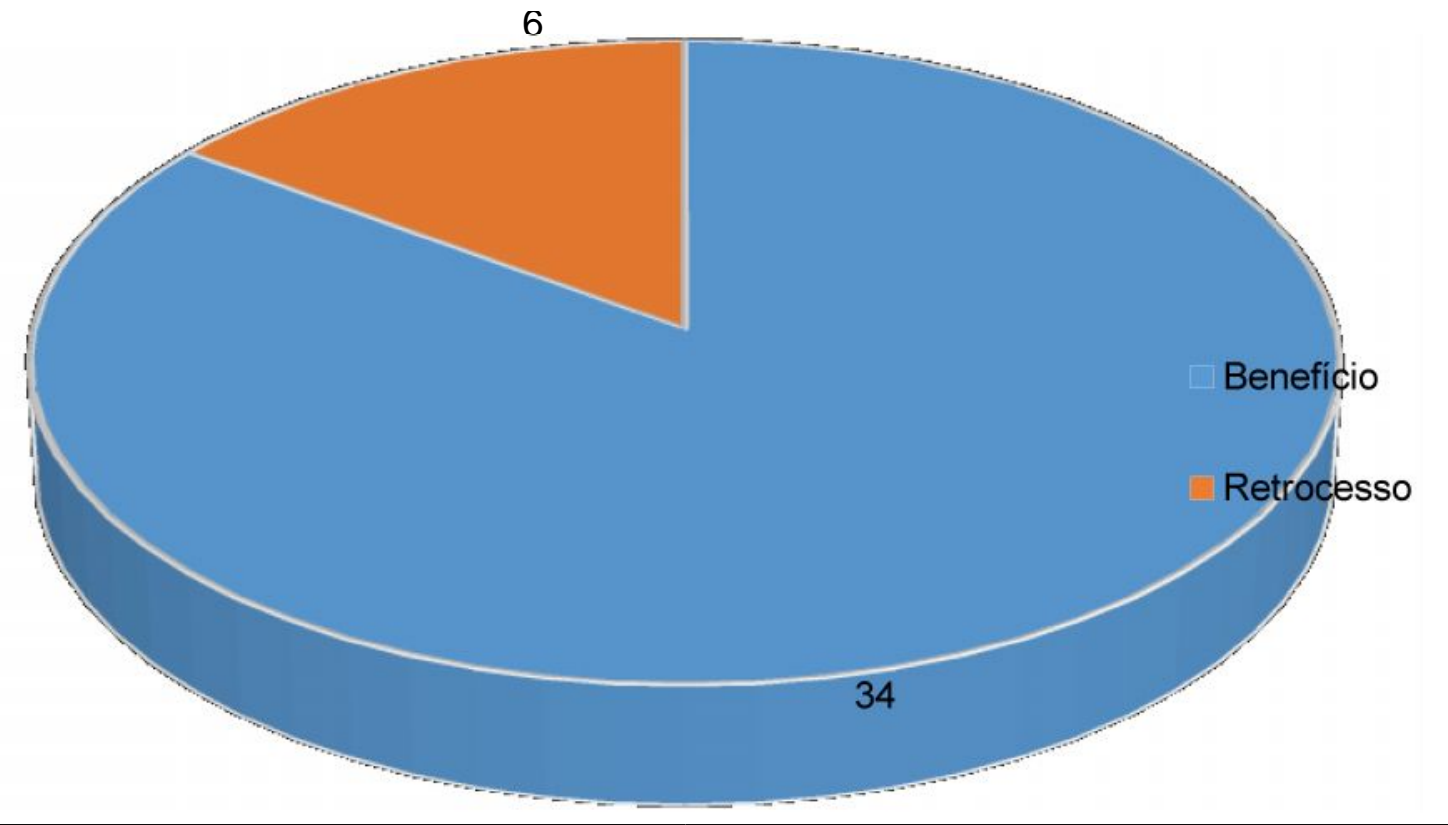

GRÁFICO 2 - Distribuição percentual sobre as considerações que os profissionais tinham em relação a integração entre Vigilância em Saúde e Atenção Básica regulamentada na nova Politica Nacional de Atenção Básica. São Mateus do Maranhão, Maranhão, Brasil, 2018.

Fonte: Dados da pesquisa (2018).

Acerca das considerações que os profissionais participantes da pesquisa tinham em relação a integração entre Vigilância em Saúde (VS) e AB regulamentada na nova PNAB, $85,0 \%$ relataram que consideravam esta nova mudança como um benefício e apenas $15,0 \%$ disseram que viam esta alteração como um retrocesso a saúde.

A PNAB de 2017 trouxe a proposta de integração das atividades especificas dos ACS e os Agentes de Combate as Endemias (ACE) com o fundamento na necessidade de agregação entre a $A B$ e a VS, na intenção das ações melhoradas em cada território. Primeiramente, a lista de funções comuns aos ACS e aos ACE será incorporada, assim sendo, o desenvolvimento de ações de promoção da saúde, prosseguimento de prevenção de agravos e riscos, realização de visitas domiciliares constantes para o monitoramento da situação de saúde da comunidade, entre outras funções, que antes eram atribuições distintas, hoje torna-se uma só (MOROSINI et al., 2018).

Em um estudo semelhante, ao presente trabalho, dirigido por Nogueira (2018) cita-se que a visão dos profissionais de saúde, principalmente quando se trata da classe afetada, são muito antagonistas a realidade. Quando referiu-se aos ACE, relataram que continuariam preservando responsabilidades sobre rotinas habituais que se referem ao seu trabalho, mas que com a mudança, o acréscimo em suas tarefas se anexariam as funções dos ACS, estes por sua vez, sofreriam uma descaracterização do seu trabalho, que já vêm perdendo espaço nas ações de educação em saúde. Tais informações estas, entraram em contradição com o 
estudo presente, já que $85,0 \%$ dos profissionais entrevistados, incluindo alguns ACS, disseram se sentirem beneficiados com tais modificações.

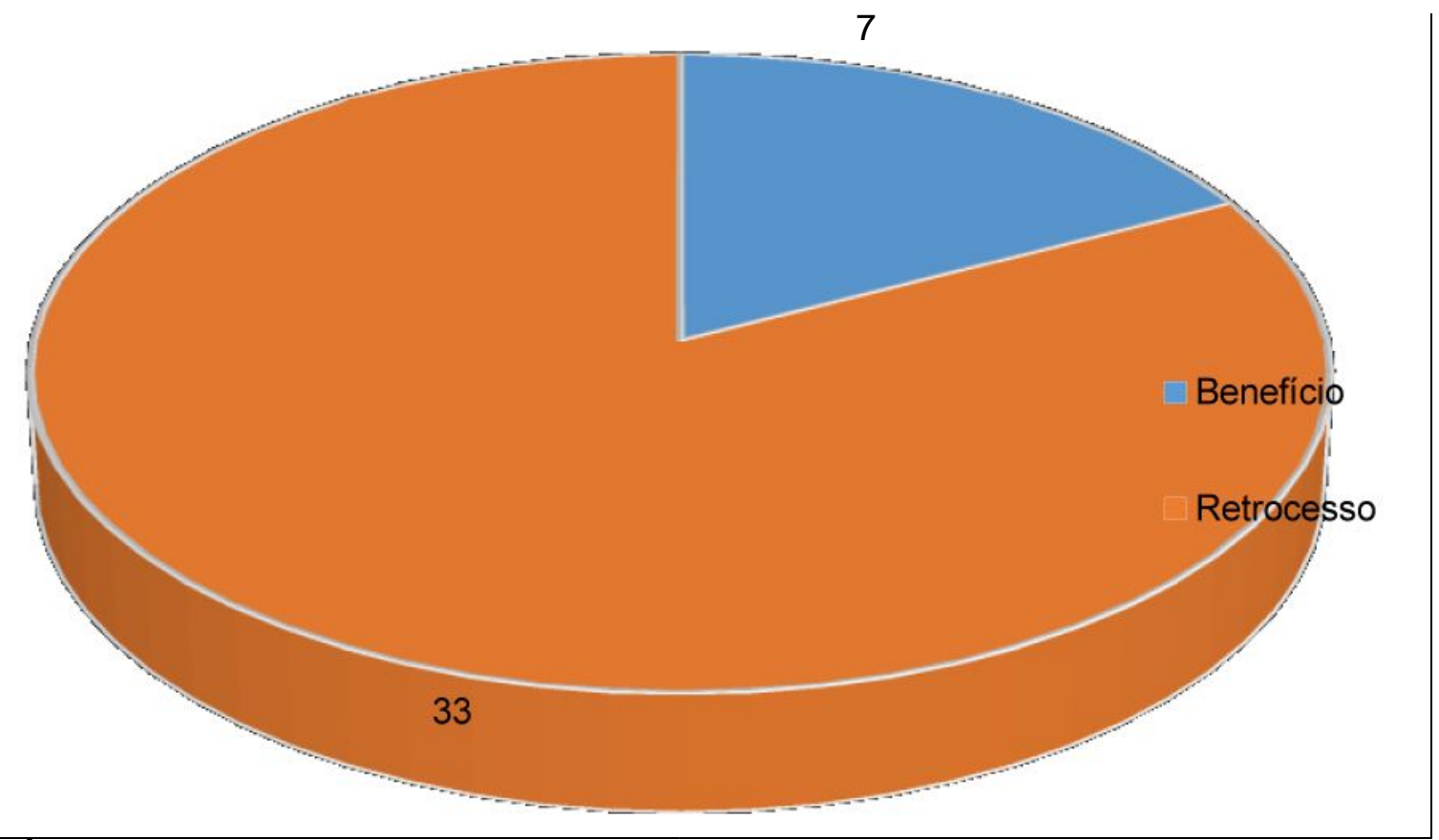

GRÁFICO 3 - Distribuição percentual sobre as considerações que os profissionais participantes da pesquisa tinham em relação ao reconhecimento das Equipes de Atenção Básica sem a obrigatoriedade de alguns profissionais. São Mateus do Maranhão, Maranhão, Brasil, 2018.

Fonte: Dados da pesquisa (2018).

A respeito das considerações que os profissionais tiveram em relação ao reconhecimento das $E A B$ sem a obrigatoriedade de alguns profissionais, a maioria $(83,0 \%)$ relataram que viram tais mudanças como um retrocesso e apenas $18,0 \%$ disseram que viram como um processo benéfico.

Em outros estudos análogos ao presente (BOAS et al., 2017; SILVA, 2018) as opiniões dos profissionais são contrárias a nova mudança, já que a falta da obrigatoriedade de alguns profissionais traz inúmeras recessões, não só para os profissionais da área da saúde, que sofreriam por sobrecarga de trabalho, devendo atender as diversas funções, além da necessidade da comunidade, para própria população, que por sua vez, padeceriam com possíveis reduções de profissionais da saúde.

Pinto (2018) disse em seu estudo que o cenário atual é lamentável, pois os dados de 2017 revelaram estagnação e até mesmo retrocesso. Em conformidade também com o levantamento feito por Santos (2017) que afirmou que tais alterações entram em contradição até mesmo com os próprios objetivos específicos da PNAB atual, quando se destacam a ampliação da $A B$ sobre as condições de saúde da população e sobre a satisfação dos seus utilizadores, pois com a nova política podese compor equipes com apenas um ACS, flexibilizando os parâmetros de cobertura da população, pelo fato do ACS ser um membro da equipe que conecta grande parte dos serviços de saúde entre a comunidade, por meio da presença constante nos territórios de abrangência, possibilitando percepções de problemas e necessidades que poderiam ser imperceptíveis aos serviços, como por exemplo, a identificação e a 
criação de possibilidades de intervenção de acordo com discernimento de cada área, contribuindo assim, na facilidade do acesso e proporcionando um vínculo estável e contínuo entre a população e os serviços de Atenção Primária a Saúde.

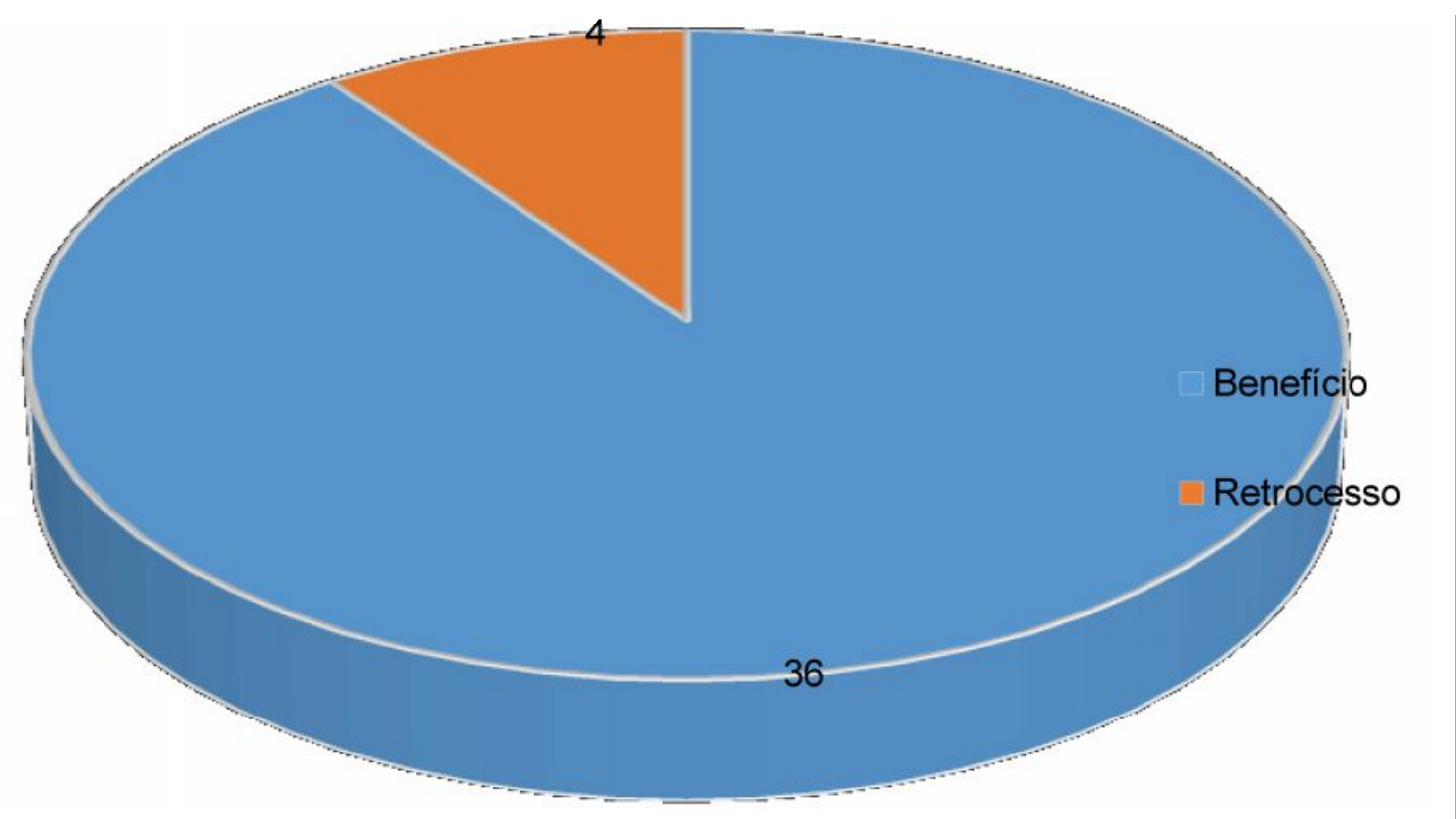

GRÁFICO 4 - Distribuição percentual sobre a visão dos profissionais participantes da pesquisa acerca da possibilidade do usuário de se vincular a mais de uma Unidade Básica de Saúde. São Mateus do Maranhão, Maranhão, Brasil, 2018.

Fonte: Dados da pesquisa (2018).

O Gráfico 4, apresenta a visão dos profissionais com relação a possibilidade do usuário de se vincular a mais de uma UBS, revelando que a superioridade $(90,0 \%)$ dos profissionais afirmaram ser benéfica tal mudança e apenas 10,0\% disseram ser um retrocesso.

A AB obtém o ofício estratégico no SUS de assegurar a universalidade do acesso, o alcance universal e a execução da integralidade, assim sendo, a $A B$ deve levar atividades de atuação a saúde o mais próximo possível das pessoas procurando resolver as questões mais relevantes de saúde e que são mais constantes (FAUSTO et al., 2014). Foi visando esse quadro que a nova PNAB por meio de mudanças, procurou superar os problemas da $A B$, principalmente depois da criação do PSF, que posteriormente veio a se tornar ESF (FACCHINI et al., 2014).

Cavalcanti et al. (2015) enumeraram em seu estudo, 10 desafios para a $A B$ no Brasil e entre estas estavam a necessidade de expansão ao acesso da população a saúde, diminuição do tempo de espera e fornecimento a atenção de qualidade, em especial, para grupos mais vulneráveis, avançando na qualidade dos serviços. Em um estudo realizado por Faria (2013) foram retratados posições e considerações de muitos profissionais da saúde atuantes na ESF, foi visto que os profissionais viam a ampliação de vínculos de UBS como uma possibilidade prática de orientação e extensão dos recursos da saúde, definindo melhorias aos modos de inscrição territorial dos serviços e as formas de acesso. 


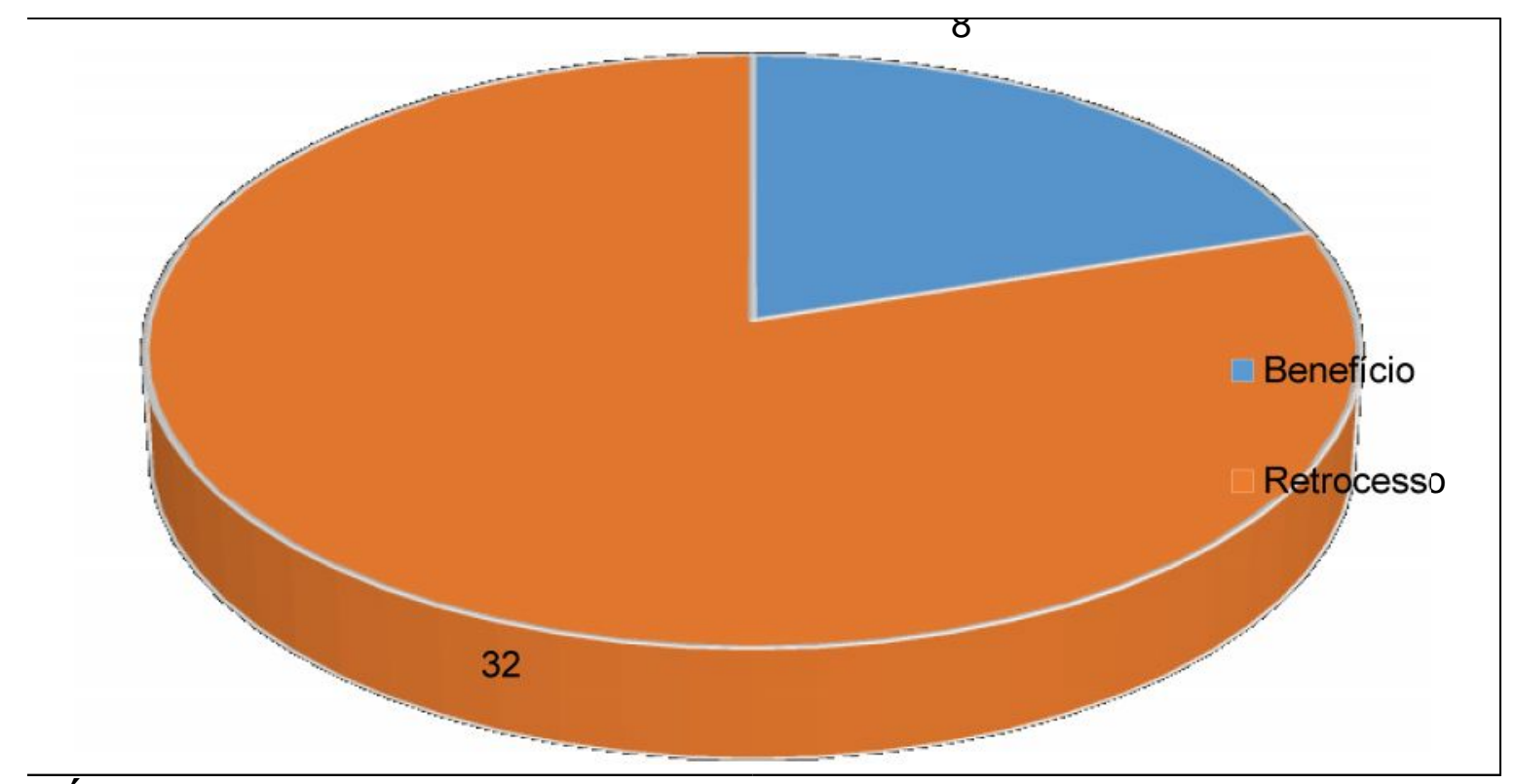

GRÁFICO 5 - Distribuição percentual sobre a visão dos profissionais participantes da pesquisa com relação a não obrigatoriedade da Estratégia Saúde da Família cobrir toda a população. São Mateus do Maranhão, Maranhão, Brasil, 2018.

Fonte: Dados da pesquisa (2018).

Em relação a opinião dos profissionais que participaram da pesquisa sobre a falta de obrigatoriedade da ESF em cobrir toda a população, os dados mostram posições bem distintas, pois a maioria $(80,0 \%)$ dos entrevistados revelaram que acham tal mudança um retrocesso para a saúde no país, já $20,0 \%$ disseram que a não obrigatoriedade de cobertura a população por parte da ESF é um benefício.

A superioridade de profissionais relatando ser um retrocesso a ausência de cobertura total a população é devido a redução de profissionais como os ACS, que são importantes para o estabelecimento de um SUS eficiente e que garante, muitas vezes, uma ligação mais expressiva com a comunidade, pois a não ser que em casos de áreas de risco e vulnerabilidade social, tal cobertura será facultativa (BOAS et al., 2017).

Em um estudo feito por Morosini et al. (2018) com profissionais da saúde pública, estes relataram que as alterações na PNAB impactariam principalmente sobre ACS, que teriam seus empregos ameaçados, consequentemente de forma linear a população, pois as novas regras, garantem em uma grande parte delas, reduções e flexibilizações deprimindo os direitos da comunidade de atendimento.

Referente ao assunto de cobertura, informações passadas pelo próprio MS revelam que a ESF alcançaria em média $58 \%$ da população, de acordo com as mudanças nos serviços, circunstâncias e ajustes nas equipes multiprofissionais, já a partir do mês de outubro de 2017, sabendo-se que tal cobertura já atingiu $100 \%$ da população em alguns municípios (PEREIRA et al., 2012).

\section{CONCLUSÃO}

A visão das mudanças e desafios que a PNAB atualizada no ano de 2017 trouxe para os profissionais da saúde que atuam na ESF em São Mateus-MA, ainda é muito controverso, em síntese, as modificações introduzidas na nova PNAB apontam para muitas direções e entre elas estão a carência de conhecimento que a população tem a respeito de seus direitos a saúde como cidadãos e a própria falta 
de conhecimento que muitos profissionais da saúde têm com relação a mudanças constitucionais. Portanto, através de vários indicativos vistos no estudo, é sugestível que novos estudos a respeito do tema sejam executados, com o intuito de apurar as inúmeras realidades e organizações da $A B$, juntamente com posicionamentos dos profissionais nos municípios do país, podendo ocasionar resultados expressivos e gerar benefícios à promoção à saúde.

\section{REFERÊNCIAS}

ABRASCO; CEBES; ENSP/Fiocruz. Associação Brasileira de Saúde Coletiva. Centro Brasileiro de Estudos de Saúde. Escola Nacional Pública Sérgio Arouca. Contra a reformulação da PNAB - nota sobre a revisão da Política Nacional de Atenção Básica. Disponível em: < https://www.abrasco.org.br/site/outras-noticias/notasoficiais-abrasco/contra-reformulacao-da-pnab-nota-sobre-revisao-da-politicanacional-de-atencao-basica/29798/>. Acesso em: 23 ago 2019.

BATALHA, E.; LAVOR, A. Nova PNAB transforma atenção básica em atenção mínima ao reduzir saúde da família e criar cesta limitada de serviços. Radis Comunicação e Saúde, n. 183, p.11-15, 2017.

BOAS, G. D. L. V.; PEREIRA, D. V. R.; SANTOS, E. K. A. A reforma da política nacional de atenção básica: mais um golpe contra o SUS. In: Anais Seminário FNCPS: Saúde em Tempos de Retrocessos e Retirada de Direitos, v.1, n.1, 2017.

BRASIL. Ministério da Saúde. Portaria no 648, de 28 de março de 2006. Aprova a Política Nacional de Atenção Básica, estabelecendo a revisão de diretrizes e normas para a organização da Atenção Básica para o Programa Saúde da Família (PSF) e o Programa Agentes Comunitários de Saúde (PACS). Brasília: Mistério da Saúde; 2006.

BRASIL. Ministério da Saúde. Portaria no 2.488, de 21 de outubro de 2011. Aprova a Política Nacional de Atenção Básica, estabelecendo a revisão de diretrizes e normas para a organização da Atenção Básica, para a Estratégia Saúde da Família (ESF) e o Programa de Agentes Comunitários de Saúde (PACS). Brasília: Ministério da Saúde; 2011.

BRASIL. Ministério da Saúde. Portaria no 2.436, de 21 de setembro de 2017. Aprova a Política Nacional de Atenção Básica, estabelecendo a revisão de diretrizes para a organização da Atenção Básica, no âmbito do Sistema Único de Saúde (SUS). Brasília: Ministério da Saúde; 2017a.

BRASIL. Ministério da Saúde. Recomendação № 035 de 11 de agosto de 2017. Reafirmam a importância da Política Nacional de Atenção Básica (PNAB), sendo fundamental para a estruturação do Sistema Único de Saúde no país. Rio de Janeiro; 2017b.

CAVALCANTI, P. C. S.; OlIVEIRA NETO, A. V.; SOUSA, M. F. Quais são os desafios para a qualificação da Atenção Básica na visão dos gestores 
municipais?. Saúde em Debate, v. 39, p. 323-336, 2015. DOI: 10.1590/0103110420151050002323.

DECLARAÇÃO DE ALMA-ATA. Conferência Internacional sobre cuidados primários de saúde; 6-12 de setembro 1978; Alma-Ata. Ministério da Saúde (BR). Secretaria de Políticas de Saúde. Projeto Promoção da Saúde. Brasília (DF): Ministério da Saúde; 2001.

FACCHINI L. A.; SILVEIRA D. S.; DURO, S. M. S.; SAES, M. O.; NUNES B. P. et al. Os sentidos da pesquisa nos processos organizativos da Estratégia Saúde da Família. In: SOUSA, M. F.; FRANCO, M. S.; MENDONÇA, A V. M. Saúde da família nos municípios brasileiros: os reflexos dos 20 anos no espelho do futuro. Campinas: Saberes; 2014. p. 844-892.

FARIA, R. M. A Territorialização da Atenção Primária à Saúde no Sistema Único de Saúde e a construção de uma perspectiva de adequação dos serviços aos perfis do território urbano. Hygeia, v. 9, n. 16, p. 121-130, 2013.

FAUSTO, M. C. R.; MENDONÇA, M. H. M.; GIOVANELLA, L. Experiências de avaliação da Atenção Básica no Brasil: notas para um debate. In: FAUSTO, M. C. R.; FONSECA, H. M. S. Rotas da Atenção Básica no Brasil: experiências do trabalho de campo do PMAQ-AB. Rio de Janeiro: Saberes Editora, p. 289-309, 2014.

GEUS, L.M M. M.; MACIEL, C. S.; BRUDA, I. C. A.; DAROS, S. J.; BATISTEL, S.; MARTINS, T. C. A.; FERREIRA, V. A.; DITTERICH, R. G. A importância na inserção do nutricionista na Estratégia Saúde da Família. Ciência \& Saúde Coletiva, v. 16, p. 797-804, 2011. DOI: 10.1590/S1413-81232011000700010.

IBGE. Instituto Brasileiro de Geografia e Estatística. Cidades. São Mateus do Maranhão. 2018. Disponível em: <https://cidades.ibge.gov.br/>. Acesso em: 23 ago 2018.

MATHIAS, M. A crise por trás da nova PNAB. Escola Politécnica de Saúde Joaquim Venâncio, 2017. Disponível em: < http://www.epsjv.fiocruz.br/noticias/reportagem/a-crise-por-tras-da-nova-pnab>.

Acesso em: 23 ago 2019.

NOGUEIRA, M. L. Impactos do neoliberalismo na política de saúde: a precarização do trabalho dos Agentes Comunitários de Saúde e a política de Atenção Básica à Saúde no Brasil. In: Convención Internacional de Salud, Cuba Salud, 2018. Disponível em: < file:///C:/Users/Usuario/Downloads/2131-4967-1-PB.pdf>. Acesso em: 23 ago 2019.

MOROSINI, M. V. G. C.; FONSECA, A. F.; LIMA, L. D. Política de Atenção Básica 2017: retrocessos e riscos para o Sistema Único de Saúde. Saúde em debate, v.42, n.116, p. 11-24, 2018. DOI: 10.1590/0103-1104201811601.

PEREIRA, A. M. M.; CASTRO, A. L. B.; OVIEDO, R. A. M.; BARBOSA, L. G.; GERASSI, C. D.; GIOVANELLA, L. Atenção primária à saúde na América do Sul em 
perspectiva comparada: mudanças e tendências. Saúde em Debate, v. 36, n. 94, p. 482-499, 2012. DOI: 10.1590/S0103-11042012000300019.

PINTO, H. A. Análise do financiamento da Política Nacional para a Atenção Básica de 1996 até 2017. Saúde em Redes, v. 4, n. 1, p. 35-53, 2018. DOI: 10.18310/2446-4813.2018v4n1p35-53.

SANTOS, K. T.; SALIBA, N. A.; MOIMAZ, S. A. S.; ARCIERI, R. M.; CARVALHO, M. L. Agente comunitário de saúde: perfil adequado a realidade do Programa Saúde da Família?. Ciência \& Saúde Coletiva, v. 16, p. 1023-1028, 2011. DOI: 10.1590/S1413-81232011000700035.

SANTOS, L. M. A percepção dos profissionais da saúde sobre os princípios da atenção primária em pequenos municípios mineiros. Revista de Administração Hospitalar e Inovação em Saúde, v.14, n.4, p.40-55, 2017. DOI: 10.21450/rahis.v14i4.4770.

SAVASSI, L. C. M. Qualidade em serviços públicos: os desafios da atenção primária. Revista Brasileira de Medicina de Família e Comunidade, v.7, n.23, p. 69-74, 2012. DOI: 10.5712/rbmfc7(23)392.

SILVA, T. P. A. O desmonte da política nacional de atenção básica (PNAB 2017) sob a forte influência do lobby dos planos de saúde suplementar no Brasil. Espaço Público, v.2, p.107-113, 2018. Disponível em: < file:///C:/Users/Usuario/Downloads/238603-124708-1-PB.pdf>. Acesso em: 23 ago 2019.

VELLOSO, A. F.; VARANDA, M. P. Difusão de inovação e atores-chave na ESF. Cadernos Saúde Coletiva, v. 25, n. 1, p.73-82, 2017. DOI: 10.1590/1414462x201700010099. 